\title{
Phase behavior of palm oil in blends with palm-based diacylglycerol
}

\begin{abstract}
Phase behavior of palm oil (PO) in blends with different concentrations (10\% intervals) of palm-based diacylglycerol oil (PO-DAG) was studied using the iso-solid diagram, solid fat content (SFC) with the hardness thermal protocol, DSC melting and crystallization curves, Xray diffraction curves, and texture analysis (hardness). Minor eutectic effects were observed at around $20-50 \%$ PO-DAG in 20-50\% SFC iso-lines. The phase behavior predicted by the iso-solid diagram as well as SFC with the hardness thermal protocol did not account for hardness variations observed between PO and PO blends with 10-40\% PO-DAG. Nevertheless, the latter could be attributed to the corresponding DSC data as well as crystal polymorphism. However, as the concentration of PO-DAG increased from $40 \%$ to $100 \%$, isoline temperatures, SFC with the hardness thermal protocol, and also hardness were found to steadily increase. PO-DAG at $10 \%$ concentration was found to have a $\beta^{\prime}$-stabilizing effect on the polymorphism of $\mathrm{PO}$, while a $\beta$-tending effect was observed as the concentration of $\mathrm{PO}$ DAG increased from $10 \%$ to $90 \%$.
\end{abstract}

Keyword: Palm-based diacylglycerol; Phase behavior; Crystallization; Polymorphism; Hardness 